\title{
Nonlinear Schwarz-Fas Methods for Unstructured Finite Element Elliptic Problems
}

J.E. Jones, P.S. Vassilevski, and C.S. Woodward

This article was submitted to the $2^{\text {nd }} M$. M.T. Conference on Computational Fluid and Solid Mechanics, Cambridge, Massachusetts, June 17-20, 2003

U.S. Department of Energy

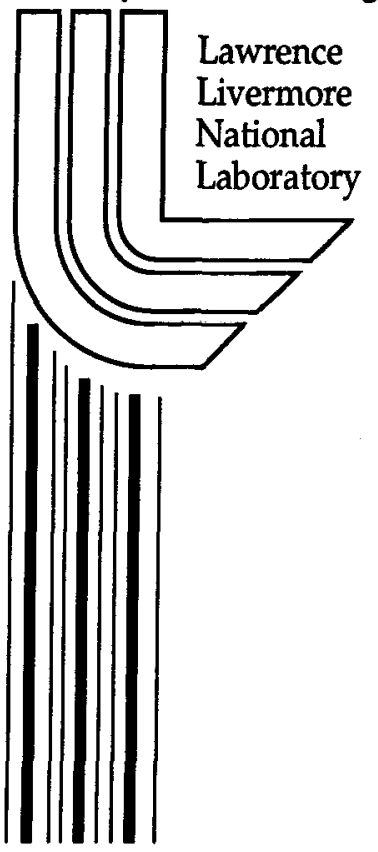

September 30, 2002 


\section{DISCLAIMER}

This document was prepared as an account of work sponsored by an agency of the United States Government. Neither the United States Government nor the University of California nor any of their employees, makes any warranty, express or implied, or assumes any legal liability or responsibility for the accuracy, completeness, or usefulness of any information, apparatus, product, or process disclosed, or represents that its use would not infringe privately owned rights. Reference herein to any specific commercial product, process, or service by trade name, trademark, manufacturer, or otherwise, does not necessarily constitute or imply its endorsement, recommendation, or favoring by the United States Government or the University of California. The views and opinions of authors expressed herein do not necessarily state or reflect those of the United States Government or the University of California, and shall not be used for advertising or product endorsement purposes.

This is a preprint of a paper intended for publication in a journal or proceedings. Since changes may be made before publication, this preprint is made available with the understanding that it will not be cited or reproduced without the permission of the author.

This report has been reproduced directly from the best available copy.

Available electronically at http://www.doc.gov/bridge

Available for a processing fee to U.S. Department of Energy

And its contractors in paper from

U.S. Department of Energy

Office of Scientific and Technical Information

P.O. Box 62

Oak Ridge, TN 37831-0062

Telephone: (865) 576-8401

Facsimile: (865) 576-5728

E-mail: reports@adonis.osti.gov

Available for the sale to the public from

U.S. Department of Commerce

National Technical Information Service

5285 Port Royal Road

Springfield, VA 22161

Telephone: (800) 553-6847

Facsimile: (703) 605-6900

E-mail: orders@ntis.fedworld.gov

Online ordering: http://www.ntis.gov/ordering.htm

OR

Lawrence Livermore National Laboratory

Technical Information Department's Digital Library

http://www.llnl.gov/tid/Library.html 


\title{
NONLINEAR SCHWARZ-FAS METHODS FOR UNSTRUCTURED FINITE ELEMENT ELLIPTIC PROBLEMS
}

\author{
JIM E. JONES, PANAYOT S. VASSILEVSKI AND CAROL S. WOODWARD
}

\begin{abstract}
This paper provides extensions of an element agglomeration AMG method to nonlinear elliptic problems discretized by the finite element method on general unstructured meshes. The method constructs coarse discretization spaces and corresponding coarse nonlinear operators as well as their Jacobians. We introduce both standard (fairly quasi-uniformly coarsened) and non-standard (coarsened away) coarse meshes and respective finite element spaces. We use both kind of spaces in FAS type coarse subspace correction (or Schwarz) algorithms. Their performance is illustrated on a number of model problems. The coarsened away spaces seem to perform better than the standard spaces for problems with nonlinearities in the principal part of the elliptic operator.
\end{abstract}

\section{INTRODUCTION}

We are interested in solving the nonlinear algebraic equations arising from finite element discretizations of nonlinear second order elliptic PDEs using finite elements. To be specific, consider the second order elliptic PDE,

$$
-\nabla \cdot(a(x, u, \nabla u) \nabla u)+g(x, u, \nabla u) u=f,
$$

posed on a polygonal domain $\Omega \in R^{2}$ with Dirichlet boundary conditions, $u=0$ on $\partial \Omega$. The functions $a=a(x, u, v)>0, g=g(x, u, v) \geq 0$, and $f=f(x)$ are given. In what follows, we assume that the functions $a, g$ and their first partial derivatives can be analytically evaluated for any value of their arguments.

The remainder of this short paper is structured as follows. We first introduce the discretization scheme, then we derive the coarse (non-inherited) nonlinear operators based on agglomeration AMGe (as proposed in [6]). Finally we formulate a standard nonlinear Schwarz -FAS algorithm for solving the resulting system of nonlinear equations exploiting coarse subspace and respective coarse nonlinear operators. The performance of the method is illustrated in the final section.

\section{Discretization}

The equation (1.1) posed variationally defines the following nonlinear operator $\mathcal{L}$

$$
(\mathcal{L} u, \varphi) \equiv \int_{\Omega}[a(x, u, \nabla u) \nabla u \cdot \nabla \varphi+g(x, u, \nabla u) u \varphi] d x
$$

Date: May 26, 2000-beginning; Today is September 30, 2002.

1991 Mathematics Subject Classification. 65F10, 65N20, 65N30.

Key words and phrases. agglomeration AMGe, subspace correction method, FAS, Schwarz, nonlinear elliptic problems, unstructured meshes, finite elements. 
Given a triangulation $\mathcal{T}=(T)$ of $\Omega$ and associated finite element space $V=V_{h}$, one can discretize $\mathcal{L}$, leading to a mapping of the form $F(\mathbf{u}) \mathbf{u}$ which can be evaluated element-wise based on the weighted element matrices,

$$
\boldsymbol{a}\left((x)_{T},(u)_{T},\left(\frac{\partial u}{\partial x}\right)_{T},\left(\frac{\partial u}{\partial y}\right)_{T}\right) A_{T}+g\left((x)_{T},(u)_{T},\left(\frac{\partial u}{\partial x}\right)_{T},\left(\frac{\partial u}{\partial y}\right)_{T}\right) G_{T} .
$$

Here $\left\{A_{T}\right\}$ and $\left\{G_{T}\right\}$ stand for the element matrices of the Laplace operator and the mass element matrices. Also, $x_{T}, u_{T}$, and $(\nabla u)_{T}$ stand for averaged values over every element $T$.

In order to compute the Jacobian of $F(\mathbf{v}) \mathbf{v}$ at $\mathbf{v}_{0}\left(v_{0}\right), J\left(\mathbf{v}_{0}\right)$, one can use the following formulas. Let, $a=a\left(v, v_{x}, v_{y}\right)$ and $g=g\left(v, v_{x}, v_{y}\right)$ and assume that one can analytically compute the partial derivatives

$$
a^{\prime}=\frac{\partial a}{\partial v}, a_{x}^{\prime}=\frac{\partial a}{\partial v_{x}}, a_{y}^{\prime}=\frac{\partial a}{\partial v_{y}}, \text { and } g^{\prime}=\frac{\partial g}{\partial v}, g_{x}^{\prime}=\frac{\partial g}{\partial v_{x}}, g_{y}^{\prime}=\frac{\partial g}{\partial v_{y}} .
$$

The corresponding formula for $J\left(\mathbf{v}_{0}\right)$, for any $\mathbf{w}$ and $\mathbf{v}$, then reads,

$$
\begin{aligned}
& \mathbf{w}^{T} J\left(\mathbf{v}_{0}\right) \mathbf{v}=\mathbf{w}^{T} F\left(\mathbf{v}_{0}\right) \mathbf{v} \quad \text { (Picard linearization) } \\
& +\left\{\sum _ { T \in \mathcal { T } } \left[(v)_{T} a^{\prime}\left(\left(v_{0}\right)_{T},\left(\frac{\partial v_{0}}{\partial x}\right)_{T},\left(\frac{\partial v_{0}}{\partial y}\right)_{T}\right)\right.\right. \\
& +\left(\frac{\partial v}{\partial x}\right)_{T} a_{x}^{\prime}\left(\left(v_{0}\right)_{T},\left(\frac{\partial v_{0}}{\partial x}\right)_{T},\left(\frac{\partial v_{0}}{\partial y}\right)_{T}\right) \\
& \left.+\left(\frac{\partial v}{\partial y}\right)_{T} a_{y}^{\prime}\left(\left(v_{0}\right)_{T},\left(\frac{\partial v_{0}}{\partial x}\right)_{T},\left(\frac{\partial v_{0}}{\partial y}\right)_{T}\right)\right] \mathbf{w}_{T}^{T} A_{T} \mathbf{v}_{0, T} \\
& +\sum_{T \in \mathcal{T}}\left[(v)_{T} g^{\prime}\left(\left(v_{0}\right)_{T},\left(\frac{\partial v_{0}}{\partial x}\right)_{T},\left(\frac{\partial v_{0}}{\partial y}\right)_{T}\right)\right. \\
& +\left(\frac{\partial v}{\partial x}\right)_{T} g_{x}^{\prime}\left(\left(v_{0}\right)_{T},\left(\frac{\partial v_{0}}{\partial x}\right)_{T},\left(\frac{\partial v_{0}}{\partial y}\right)_{T}\right) \\
& \left.\left.+\left(\frac{\partial v}{\partial y}\right)_{T} g_{y}^{\prime}\left(\left(v_{0}\right)_{T},\left(\frac{\partial v_{0}}{\partial x}\right)_{T},\left(\frac{\partial v_{0}}{\partial y}\right)_{T}\right)\right] \mathbf{w}_{T}^{T}, G_{T} \mathbf{v}_{0, T}\right\} .
\end{aligned}
$$

Here, we need averaged values $(.)_{T}$ of any vector (function) and its derivatives.

\section{Coarsening}

A coarse nonlinear operator $\mathcal{L}_{H}$, for a coarse finite element space $V_{H}$ (constructed by $\mathrm{AMGe}$, in the form proposed in Jones and Vassilevski [6], for example) is defined, for $u, \varphi \in V_{H}$, by

$$
\begin{aligned}
\left(\mathcal{L}_{H} u, \varphi\right) \equiv & \sum_{T \in \mathcal{T}_{H}}\left[a\left(x_{T}, u_{T},(\nabla u)_{T}\right) \int_{T} \nabla u \cdot \nabla \varphi d x\right. \\
& \left.+g\left(x_{T}, u_{T},(\nabla u)_{T}\right) \int_{T} u \varphi d x\right]
\end{aligned}
$$

where $x_{T}, u_{T}$, and $(\nabla u)_{T}$ are averaged values over every element $T$. In matrix-vector form this reads, for $u, \varphi \in V_{H}$, and their respective coefficient vectors restricted to any element $T, \mathbf{u}_{T}$ and $\underline{\varphi}_{T}$,

$$
\left(\mathcal{L}_{H} u, \varphi\right) \equiv \sum_{T \in \mathcal{T}_{H}} \mathbf{u}_{T}^{T}\left(a\left(x_{T}, u_{T},(\nabla u)_{T}\right) A_{T}+g\left(x_{T}, u_{T},(\nabla u)_{T}\right) G_{T}\right) \underline{\varphi}_{T} .
$$


Here $\left\{A_{T}\right\}$ and $\left\{G_{T}\right\}$ stand for the coarse element matrices of the Laplace operator and the mass element matrices.

Thus one needs an element averaging procedure $(\cdot)_{T}$ on all grids.

3.1. Computing derivatives on coarse grids by element averaging. The following simple element-wise approximations to the derivatives are feasible,

$$
\begin{aligned}
& \left.\frac{\partial u}{\partial x}\right|_{T} \simeq \frac{1}{|T|} \mathbf{X}^{T} A_{T} \mathbf{u},\left.\quad \frac{\partial u}{\partial y}\right|_{T} \simeq \frac{1}{|T|} \mathbf{Y}^{T} A_{T} \mathbf{u}, \\
& \left.\frac{\partial u}{\partial z}\right|_{T} \simeq \frac{1}{|T|} \mathbf{Z}^{T} A_{T} \mathbf{u} .
\end{aligned}
$$

This is motivated by the equalities,

$$
\mathbf{X}^{T} A_{T} \mathbf{u}=\int_{T} \nabla u \cdot \nabla x=\int_{T} \nabla u \cdot\left(\begin{array}{l}
1 \\
0 \\
0
\end{array}\right)=\left.\int_{T} \frac{\partial u}{\partial x} \simeq|T| \frac{\partial u}{\partial x}\right|_{T} .
$$

The relation (" $\simeq$ ") is actually equality if $u$ is linear over $T$.

Similarly, one can perform element averaging of $u$ based on $G_{T}$,

$$
\left.u\right|_{T} \simeq \frac{1}{|T|} \int_{T} 1 . u d x=\frac{1}{|T|}(\mathbf{1})^{T} G_{T} \mathbf{u}_{T}
$$

Here, $X, Y, Z$ and 1 stand for the vector representations of the linear functions $x, y, z$, and 1 .

\section{SCHWARZ-FAS AMGE ALGORITHM}

Consider the fine grid nonlinear problem

$$
F(\mathbf{u}) \mathbf{u}=\mathbf{f},
$$

and let $\mathbf{u}_{0}$ be a given initial approximation. Also, let the coarse subspaces $\mathbf{V}_{k}$ and the respective interpolation matrices $P_{k}: \mathrm{V}_{k} \mapsto \mathrm{V}$ be given. Finally, let $I_{k}$ be a simple (injection) operator which restricts a fine-grid vector to a coarse grid one in $\mathbf{V}_{k}$.

Then one performs the following steps to get a next approximation to $\mathbf{u}$.

Algorithm 4.1 (Subspace Correction-FAS Method).

- For $k=1, \ldots, p$ loop over the coarse subspaces $V_{k}$ :

(1) restrict global residual $\mathbf{r}=\mathbf{f}-F(\mathbf{u}) \mathbf{u}$ to $\mathbf{V}_{k}$, i.e., $\mathbf{r}_{k}=P_{k}^{T} \mathbf{r}$.

(2) solve (e.g., using Newton's method) the coarse nonlinear problem

$$
F_{k}\left(\mathbf{u}_{k}\right) \mathbf{u}_{k}=\mathbf{f}_{k},
$$

with an initial approximation $\mathbf{u}_{k}^{0}=I_{k} \mathbf{u}$ and a right-hand side $\mathbf{f}_{k}=\mathbf{r}_{k}+$ $F_{k}\left(\mathbf{u}_{k}^{0}\right) \mathbf{u}_{k}^{0}$.

(3) interpolate the coarse grid correction $\mathbf{u}_{k}-\mathbf{u}_{k}^{0}$ and update the fine-grid approximation; that is,

$$
\mathbf{u}:=\mathbf{u}+P_{k}\left(\mathbf{u}_{k}-\mathbf{u}_{k}^{0}\right) .
$$


- an optional "post-smoothing" step; for example, a few nonlinear Gauss-Seidel iterations.

- the new nonlinear FAS iterate is $\mathbf{u}:=\mathbf{u}+\underline{\xi}$.

For details on theory regarding the structured finite element case, cf. Dryja and Hackbusch [5], Xu [11], Tai and Espedal [9], Tai and Xu [10], and for the classical FAS method, cf. Brandt [3] and Briggs et al. [4].

For some other multigrid approaches for nonlinear diffusion equations on unstructured meshes, cf., Mavriplis [7].

\section{A NONLINEAR TEST PROBLEM}

Consider a nonlinear elliptic problem with more general nonlinearity:

$$
-\nabla \cdot(a(u, \nabla u) \nabla u)+u^{3}=f,
$$

in $\Omega=(0,1)^{2}$ with Dirichlet boundary conditions. Here, $a(u, \nabla u)=\frac{1}{\sqrt{\epsilon+u^{2}+\mid \overline{\left.\nabla u\right|^{2}}}}, \epsilon=$ 0.001 . The r.h.s. function $f$ is chosen such that $u=x(1-x) y(1-y)$ is the exact solution.

The initial iterates for the FAS subspace correction method were chosen 0.9 times the true solution. The iterations are terminated after relative residual $\ell^{2}$-norm reduction of the initial residual by a factor of $10^{-6}$ has been achieved.

5.1. Coarsened away meshes. The coarse spaces corresponded to our AMGeconstructed ones. The coarse agglomerated elements are obtained by first partitioning the initial set of fine elements using METIS into "\# domains" subsets, then the elements in a given subdomain were selected (fixed) allowing for agglomeration only the elements that are more than one layer away from the fixed set of elements on the previous level. Thus one ends up with algebraically constructed finite element spaces of small dimension but of global nature. This is illustrated in Fig. 1 and Fig. 2.

Then the thus constructed coarse spaces, operators and their Jacobians are used in Algorithm 4.1 and in a non-linear preconditioned GCG method (cf. [1]) where the fine-grid Jacobian is preconditioned with additive Schwarz preconditioner coming from the coarsened away spaces. The results are found in Tables 1 and 2 .

\section{REFERENCES}

[1] O. AXELSSON AND A. T. ChRONOPOUlós, "On non-linear generalized conjugate gradient methods", Numerische Mathematik 69(1994), pp. 1-15.

[2] R. E. Bank and M. Holst, "A NEW PARADIGM FOR PARAllel ADAPTive MESHING ALGORITHMS", SIAM Journal on Scientific Computing 22(2000), pp. 1411-1443.

[3] A. BRANDT, "Multi-level adaptive solutions to boundary-value problem" Math. Comp. 31(1977), pp. 333-390.

[4] W. L. Briggs, V. E. Henson, and S. F. McCormick, "A Multigrid tutorial", SIAM, Philadelphia, PA, 2000.

[5] M. DRYJA AND W. HACKBUSCH, "On the nonlinear domain decomposition method", BIT 37(1997), pp. 296-311.

[6] J. E. JONES AND P. S. VASSILEVSKI, "AMGe based on element agglomerations", SIAM J. Scientific Computing 23(2001), pp. 109-133. 
[7] D. J. MAVRIPLIS, "Multigrid approaches to non-linear diffusion problems on unstructured meshes", Numer. Lin. Alg. Appl. 8(2001), pp. 499-512.

[8] "METIS: Family of multilevel partitioning algorithms", available at: http://wwwusers.cs.umn.edu/-karypis/metis/.

[9] X.-C. TAI AND M. ESPEDAL, "Rate of convergence of some space decomposition methods for linear and non-linear problems", SIAM J. Numer. Anal. 35(1998), pp. 1558-1570.

[10] X.-C. TAI AND J. XU, "Global and uniform convergence of subspace correction methods for some convex optimization problems", Math. Comp. 71(2002), pp. 105-124.

[11] J. Xu, "Two-grid discretization techniques for linear and non-linear PDEs", SIAM J. Numer. Anal. 33(1996), pp. 1759-1777. 
JIM E. JONES, PANAYOT S. VASSILEVSKI AND CAROL S. WOODWARD

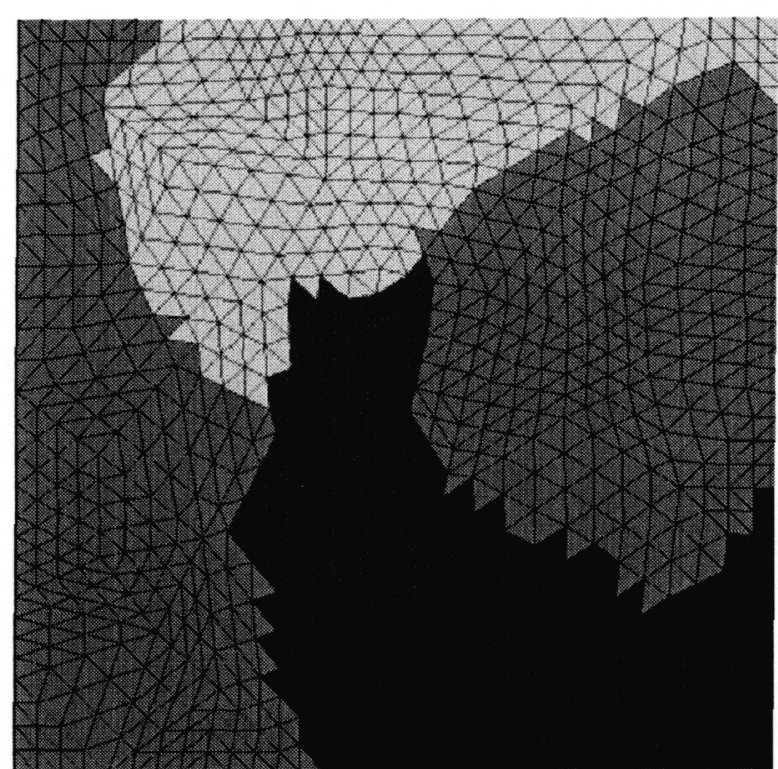

FIGURE 1. Fine mesh partitioned into four mesh subdomains: 1,600 fine elements, 2460 fine degrees of freedom. Each subdomain consists of 400 elements and is represented by a single color. 


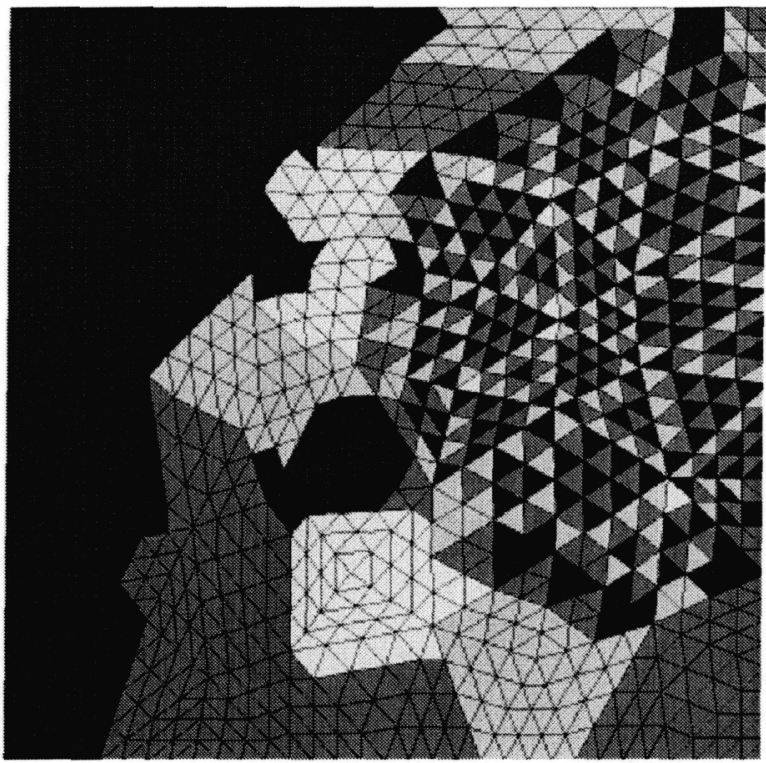

FIGURE 2. An agglomeration based coarsened away mesh: 1,600 fine elements, 466 agglomerated elements, 400 subdomain elements, 270 coarse degrees of freedom. Each color represents an agglomerate. 
TABLE 1. Unstructured triangular grids; number of iterations of FAS subspace correction method with smoothing.

\begin{tabular}{|c||c|c|c|c|}
\hline \# domains & 400 elements & 1600 elements & 6400 elements & 25600 elements \\
\hline \hline 4 & 10 & 10 & 12 & 14 \\
\hline 8 & 9 & 11 & 16 & 17 \\
\hline 16 & 12 & 15 & 15 & $\star$ \\
\hline
\end{tabular}

TABLE 2. Unstructured triangular grids; number of iterations of preconditioned nonlinear GCG method. Each second row shows the total number of preconditioned (with additive Schwarz Jacobian preconditioner) GCG iterations for inexact solving with fine-grid Jacobians for achieving relative tolerance 0.0001 .

\begin{tabular}{|c||c|c|c|c|}
\hline \# domains & 400 elements & 1600 elements & 6400 elements & 25600 elements \\
\hline \hline 4 & 3 & 3 & 3 & 4 \\
& 47 & 67 & 92 & 196 \\
\hline 8 & 3 & 3 & 3 & 3 \\
& 59 & 72 & 102 & 136 \\
\hline 16 & 3 & 3 & 3 & 4 \\
& 73 & 100 & 149 & 204 \\
\hline
\end{tabular}

Center for A pplied Scientific Computing, UC lawrence livermore National labOratory, P.O. Box 808, L-560, Livermore, CA 94551, U.S.A.

E-mail address: jjones@llnl.gov, vassilevskil@llnl.gov, cswoodward@llnl.gov 